\title{
Hepatitis E virus in hematopoietic stem cell transplant recipients: A systematic review
}

\author{
Sara Cruz ${ }^{\mathrm{a}}$, Carla Campos ${ }^{\mathrm{b}}$, Mafalda Timóteo $^{\mathrm{a}}$, Ana Tavares ${ }^{\mathrm{c}}$, Maria São José Nascimento ${ }^{\mathrm{d}, \mathrm{e}}$, \\ Rui Medeiros ${ }^{\mathrm{a}, \mathrm{f}, \mathrm{g}}$, Hugo Sousa ${ }^{\mathrm{a}, \mathrm{f}, \mathrm{h}, \mathrm{i}, *}$
}

${ }^{a}$ Molecular Oncology and Viral Pathology Group (CI-IPOP), Portuguese Oncology Institute of Porto, Rua Dr. António Bernardino de Almeida, 4200-072, Porto, Portugal

${ }^{\mathrm{b}}$ Microbiology Service, Portuguese Oncology Institute of Porto, Rua Dr. António Bernardino de Almeida, 4200-072, Porto, Portugal

c Pathology Department, Portuguese Oncology Institute of Porto, Rua Dr. António Bernardino de Almeida, 4200-072, Porto, Portugal

${ }^{\mathrm{d}}$ Microbiology Laboratory, Biological Sciencies Department, Pharmacy Faculty, University of Porto, 4050-313, Porto, Portugal

${ }^{\mathrm{e}}$ Epidemiology Research Unit, Public Health Institute, University of Porto, 4050-313, Porto, Portugal

${ }^{\mathrm{f}}$ Virology Service, Portuguese Oncology Institute of Porto, Rua Dr. António Bernardino de Almeida, 4200-072, Porto, Portugal

${ }^{\mathrm{g}}$ Research Department, Portuguese League Against Cancer (Liga Portuguesa Contra o Cancro-Núcleo Regional do Norte), Estrada interior da Circunvalação 6657, 4200-

172, Porto, Portugal

${ }^{\mathrm{h}}$ Life and Health Sciences Research Institute (ICVS), School of Health Sciences, University of Minho, Campus de Gualtar, 4710-057, Braga, Portugal

${ }^{\mathrm{i}}$ ICVS/3B's - PT Government Associate Laboratory, Braga/Guimarães, Portugal

\section{A R T I C L E I N F O}

\section{Keywords:}

Hepatitis E virus

HEV

Stem cell transplantation

HSCT

Prevalence

Systematic review

\begin{abstract}
A B S T R A C T
Background: In developed countries, Hepatitis E virus (HEV) infections, especially by HEV-3, are frequently associated with asymptomatic infection or self-limiting acute hepatitis, although it has been described as a cause of chronic infection, especially in immunocompromised hots. Hematopoietic stem cell transplant (HSCT) recipients have been recognized as an important risk group for HEV infection due to their prolonged immunosuppression state.

Objectives: We aimed to perform a systematic review of published data to evaluate HEV infection prevalence among HSCT recipients.

Study Design: Literature search was performed concerning published manuscripts regarding 'hepatitis E virus AND stem cell transplantation' following the Preferred Reporting of Systematic Reviews and MetaAnalyses (PRISMA) guidelines. Statistical analysis was performed using the MetaXL software to estimate the overall prevalence of HEV infection according to the different diagnostic approaches (HEV RNA and anti-HEV IgM and/ or IgG detection).

Results: A total of 7 manuscripts were included for data analysis, with 6 studies performed in Europe and 1 study in China. Regarding HEV RNA detection, the overall HEV infection prevalence was 1.50\% (95\% CI: 0.70-2.60). The overall anti-HEV IgM seroprevalence was 2.00\% (95\% CI: 0.30-4.50), and anti-HEV IgG was $11.4 \%$ (95\% CI: 1.80-26.3).

Conclusions: This systematic review reveals that the overall prevalence of HEV infection in HSCT patients differ according to the diagnostic, thus emphasizing the need of more studies to increase the data regarding prevalence and incidence in HSCT recipients.
\end{abstract}

\section{Background}

Hepatitis E virus (HEV) was recently recognized as the most common cause of acute viral hepatitis worldwide, with the World Health Organization (WHO) estimating 20 million infections, $>3$ million acute cases, and $>57,000$ HEV-related deaths, annually [1,2]. In industrialized countries, hepatitis $\mathrm{E}$ was considered a rare disease until the discovery of the new HEV genotypes that turned this infection a concern of public health. In fact, four major HEV genotypes infect humans: genotypes 1 (HEV-1) and 2 (HEV-2), that are transmitted through the fecal-oral route via fecal contaminated water, being prevalent in areas of poor sanitation, such as in developing countries [3,4]; and genotypes 3 (HEV-3) and 4 (HEV-4) that are zoonotic viruses transmitted to humans mainly through undercooked pork and boar meat and

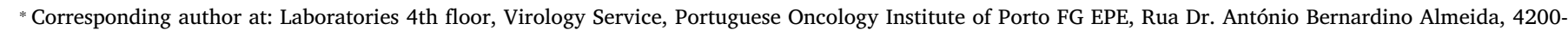
072, Porto, Portugal.

E-mail address: hugo.sousa@ipoporto.min-saude.pt (H. Sousa). 
contact with pigs [3-8], although, the transfusion of blood products has also been recently recognized as a risk factor [9-11]. HEV-3 is today recognized as the main cause of sporadic autochthonous cases in industrialized countries $[3,4]$ and the numbers show that the incidence of reported cases has been increasing [12-17].

In Europe, HEV infections are mainly caused by HEV-3, a genotype that causes asymptomatic infection or self-limiting acute hepatitis in healthy individuals, although it can lead to chronic infection with rapidly progressive cirrhosis in immunosuppressed patients, such as individuals with HIV, hematological malignancies, or transplant-related patients [18-21]. Solid organ transplant (SOT) recipients and hematopoietic stem cell transplant (HSCT) recipients are an important group at risk for HEV infection due to their prolonged immunosuppression state, that increases the risk for developing chronic infection [19]. Patients undergoing allogeneic-HSCT (allo-HSCT) have in general a higher risk for viral infections and higher incidence of graft-versus-host disease (GVHD) than autologous-HSCT (auto-HSCT) [22,23]. In allo-HSCT recipients, progression to chronic infection may be favored by the severity of immunosuppression, which results in impaired immune reconstitution, including insufficient lymphocyte recovery, that are risk factors for post-transplantation infections [24-26]. Moreover, HSCT recipients have a high transfusion burden, which is a problem particularly in countries that have not introduced HEV screening in blood donations $[22,27,28]$.

The evidence that most of the HEV-3 infections become chronic in immunocompromised hosts, especially those after transplantation, makes these patients an important group of study [29,30]. In SOT recipients, the reported acute HEV infection prevalence is $1-3 \%$, with $47-83 \%$ of the patients developing chronic hepatitis [31-33], however, the prevalence and incidence of HEV in HSCT recipients is largely unknown.

\section{Objectives}

In the present study, we aim to summarize published data regarding HEV infections in HSCT recipients by performing a systematic review of the literature.

\section{Study Design}

\subsection{Literature search and study selection}

Preferred Reporting of Systematic Reviews and MetaAnalyses (PRISMA) guidelines were followed in the preparation of this systematic review. Different queries, including MeSH terms, were tested and the literature search was performed with the query that obtained more representative manuscripts: 'hepatitis E virus AND stem cell transplantation'. PubMed and Scopus databases were searched, independently by two of the authors (SC and CC), for published manuscripts on $31^{\text {st }}$ January 2019 without restrictions on time period, sample size or population.

The eligibility criteria applied to studies were: 1) HEV infection (present or past) identified by the presence of HEV RNA and/or HEVspecific antibodies ( $\operatorname{IgM} / \mathrm{IgG})$ in tested samples; 2) HSCT recipients; 3) provide prevalence data. The exclusion criteria applied were: 1) duplicate data; 2) other types of manuscripts (reviews, case reports, comments or letters to the editor); 3) no access to abstracts and/or full texts; and 4) other languages rather than English, Spanish or Portuguese. Manuscript titles and abstracts were screened according to the eligibility criteria and selected manuscripts were fully reviewed for data extraction (author, publication date, country, population, age range, type of HSCT, HEV detection methods and the number of positive and negative cases). All manuscripts were reviewed independently by two of the authors (SC and $\mathrm{CC}$ ) with disagreements mediated by the senior researcher (HS).

\subsection{Statistical analysis}

All data was inserted in a database that was used for prevalence analysis and comparison between studies. Prevalence analysis was performed using the MetaXL program version 5.3 (EpiGear International, Sunrise Beach, Queensland, Australia). The overall prevalence of HEV infection was estimated using the different approaches of diagnosis (HEV RNA or anti-HEV IgM/IgG detection) in HSCT recipients pooling the study data using the random effects model. The random effects model was used since a considerable heterogeneity among studies was expected, due largely to the different settings (populations, types of patients, age, gender, diagnostic methods) in which studies were conducted. The double arcsine transformation method was used for variance stabilization [34] considering a 95\% confidence interval and a 5\% statistical significance level $(p<0.050)$.

\section{Results}

\subsection{Study selection and description}

The literature search retrieved a total of 73 manuscripts from both databases, and after duplicates removal, a total of 54 records were screened (Fig. 1). After applying inclusion/exclusion criteria, 41 records were excluded: language $(n=1)$, reviews $(n=18)$, other types of articles such as Case Reports and Letter to the Editor $(n=9)$, and studies not related to HSCT patients or HEV detection $(n=13)$. A total of 13 full-text articles were assessed for full-reading of which 6 were excluded: 1 review, 4 case reports and 1 was not performed in HSCT patients. The bibliography of the selected manuscripts was reviewed to identify any new publications and no other article was added to the analysis.

After the full revision process, we included 7 manuscripts for data analysis [18,21,35-39] (Table 1). Overall, these 7 studies included a total of 1178 HSCT patients from different countries: six studies were performed in Europe (United Kingdom, Netherlands, France, and Germany) $[18,21,36,36,37,38,39]$ and one study in China 35]. These studies evaluated the prevalence of HEV infection based on the detection of HEV RNA $(n=7)$ and/or the presence of anti-HEV $\operatorname{IgM}(n=3) / \operatorname{IgG}$ $(n=4)$. Phylogenetic analysis was performed in only three of these seven studies, revealing only HEV-3 genotype [21,37,39].

Here, we briefly resume the data from all included studies. In China, a study evaluated HEV infection in 177 haploidentical-HSCT recipients that presented unexplained elevated transaminases after transplant, identifying 7 patients with an acute/current HEV infection (2 positives for HEV RNA and 5 positives for anti-HEV IgM/IgG) [35]. In the United Kingdom, two studies identified a total of 4 recipients with an acute HEV infection based in the presence of HEV RNA: in one study, 259 HSCT recipients (111 allo-HSCT, 145 auto-HSCT, and 3 CD34 top-up procedures) were evaluated and only 1 HEV RNA positive patient was identified [39]; while the other study analyzed 144 allo-HSCT recipients and 3 patients were positive for HEV RNA [21]. In the Netherlands, two studies analysed HEV infection in HSCT: one study was performed in 130 allo-HSCT recipients with elevated alanine aminotransferase (ALT), identifying 5 HEV RNA positive patients [36]; the other study analyzed 328 allo-HSCT recipients transplanted over a 5year period, identifying a total of 10 patients with a current infection (8 were positive for HEV RNA and 2 were positive for anti-HEV IgM) and 41 patients were seropositive for anti-HEV IgG [37]. In France, a study with 88 HSCT recipients (72 allo-HSCT and 16 auto-HSCT) revealed that none patient tested positive for HEV RNA, while 3 were positive for anti-HEV IgM [18]. Interestingly, this study found a seroprevalence of anti-HEV IgG of $125 \%(11 / 88)$ and $364 \%(32 / 88)$ in the same group of HSCT recipients when using two different enzyme immunoassays (EIA) methods. In the study from Germany with 52 allo-HSCT recipients with elevated ALT, but without any specific cause of hepatitis, none of them tested positive for HEV RNA, while 3 were positive for anti-HEV IgG 


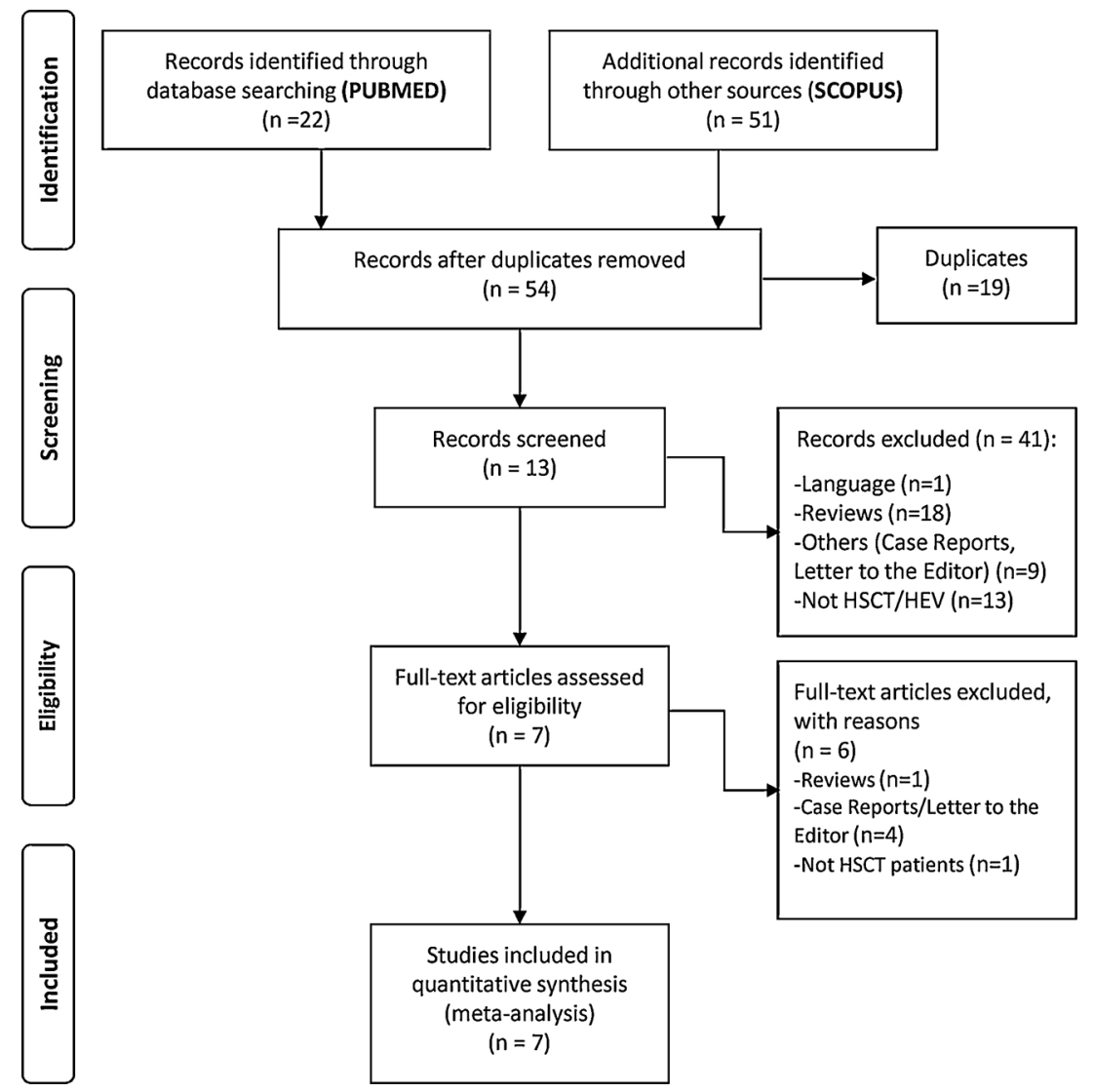

Fig. 1. PRISMA flow diagram.

Abbreviations: HEV, Hepatitis E Virus; HSCT, Hematopoietic stem cell transplantation.

[38].

\subsection{HEV infection prevalence analysis}

We have performed an analysis of the prevalence of HEV infection in HSCT recipients according to the different approaches of diagnosis. The diagnosis of HEV positive cases is based on the identification of a patient with an HEV RNA positive sample (serum, plasma, blood or feces) or both anti-HEV IgM and IgG positive sample, nevertheless, the seroprevalence of the infection is referred to the detection of anti-HEV IgG only.

Considering the analysis of HEV RNA detection, a total of 1178
HSCT patients were studied and only 19 were positive, which gives an overall prevalence of $1.50 \%$ (95\% CI: $0.70-2.60$ ) with no significant difference observed between the different studies ( $p=0.090$; Fig. 2).

The analysis of anti-HEV IgM and IgG was performed separately: the detection of anti-HEV IgM as marker of acute infection was tested in a total of 593 HSCT patients with only 10 positive cases, giving an overall anti-HEV IgM prevalence of $2.00 \%$ (95\% CI: $0.30-4.50 ; p=0.060$; Fig. 3); while the detection of anti-HEV IgG, a marker of past infection, was described in a total of 645 samples, 82 HSCT recipients were found positive to anti-HEV IgG giving an overall IgG seroprevalence of $11.4 \%$ (95\% CI: 1.80-26.3), with statistically significant differences between the studies ( $p<0.001$; Fig. 4).

Table 1

Details of the studies reporting HEV infection in HSCT recipients used in data analysis.

\begin{tabular}{|c|c|c|c|c|c|c|}
\hline First author (country, year) & Patients, $\mathrm{n}$ & Type of HSCT & HEV Diagnostic Methods & Assay & Positive, $\mathrm{n}$ & Prevalence \\
\hline \multirow[t]{3}{*}{ Tang FF et al. (China, 2019) } & \multirow[t]{3}{*}{177} & \multirow[t]{3}{*}{ Allo-HSCT } & Anti-HEV IgM & EIA (MP Diagnostics ${ }^{\circledR}$ ) & 5 & $2.82 \%$ \\
\hline & & & Anti-HEV IgG & & & \\
\hline & & & HEV RNA & Commercial RT-qPCR & 2 & $1.13 \%$ \\
\hline Reekie I, et al. (England, 2018) & 259 & Allo/Auto-HSCT & HEV RNA & In-house RT-qPCR & 1 & $0.39 \%$ \\
\hline Ankcorn MJ, et al. (United Kingdom, 2018) & 144 & Allo-HSCT & HEV RNA & In-house RT-qPCR & 3 & $2.08 \%$ \\
\hline Willemse SB et al. (Netherlands, 2017) & 130 & Allo-HSCT & HEV RNA & Commercial RT-qPCR & 5 & $3.85 \%$ \\
\hline \multirow[t]{3}{*}{ Verluis J, et al. (Netherlands, 2013) } & \multirow[t]{3}{*}{328} & \multirow[t]{3}{*}{ Allo-HSCT } & Anti-HEV IgM & EIA (Wantai $\left.{ }^{\circledast}\right)$ & 2 & $0.61 \%$ \\
\hline & & & Anti-HEV IgG & & 41 & $12.5 \%$ \\
\hline & & & HEV RNA & In-house RT-qPCR & 8 & $2.44 \%$ \\
\hline \multirow[t]{4}{*}{ Abravanel F, et al. (France, 2012) } & \multirow[t]{4}{*}{88} & \multirow[t]{4}{*}{ Allo/Auto-HSCT } & Anti-HEV IgM & EIA (Adaltis ${ }^{\circledast}$ ) & 3 & $3.41 \%$ \\
\hline & & & Anti-HEV IgG & & 11 & $12.5 \%$ \\
\hline & & & Anti-HEV IgG & EIA (Wantai ${ }^{\circledast}$ ) & 32 & $36.4 \%$ \\
\hline & & & HEV RNA & In-house RT-qPCR & 0 & $0.00 \%$ \\
\hline \multirow[t]{2}{*}{ Koenecke C, et al. (Germany, 2012) } & \multirow[t]{2}{*}{52} & \multirow[t]{2}{*}{ Allo-HSCT } & Anti-HEV IgG & EIA (Abbott ${ }^{\circledR}$ ) & 3 & $5.77 \%$ \\
\hline & & & HEV RNA & In-house Nested RT-PCR & 0 & $0.00 \%$ \\
\hline
\end{tabular}

Abbreviations: EIA, Enzyme Immunoassay; HEV, Hepatitis E Virus; HSCT, Hematopoietic stem cell transplantation; IgG, Immunoglobulin G; IgM, Immunoglobulin M; n, sample size; Nested RT-PCR, Nested Reverse Transcription-Polymerase Chain Reaction; RT-qPCR, Reverse Transcription-quantitative Polymerase Chain Reaction. 


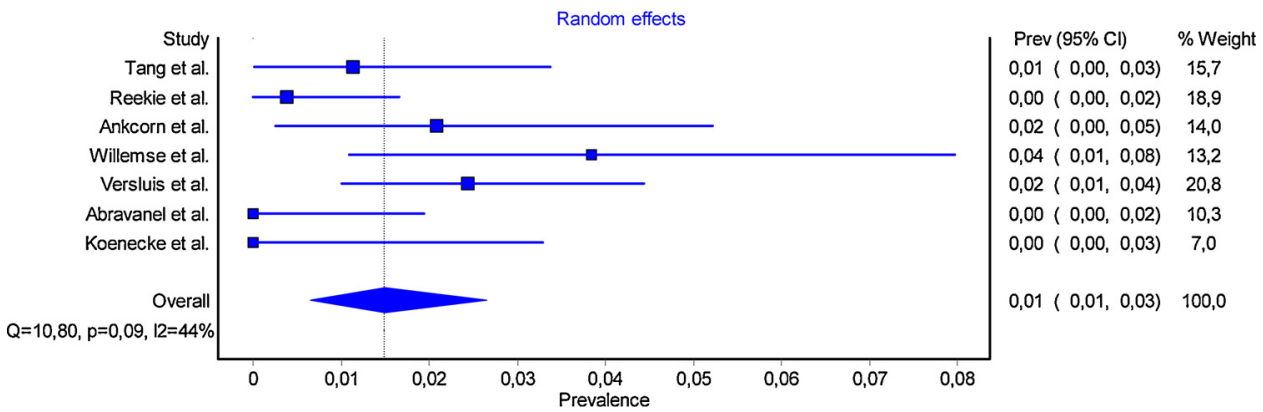

Fig. 2. Forest plot of the overall prevalence based on the detection of HEV RNA.

Abbreviations: p, $p$-value; Prev (95\%CI), Prevalence 95\% Confidence Interval.

\section{Discussion}

The evidence that most of the HEV-3 infections become chronic in immunocompromised hosts, especially those after transplantation, makes these patients an important group of study [29,30]. Since the prevalence and incidence of HEV in HSCT recipients is largely unknown, we have performed a systematic review to understand the burden of HEV infection in these group of immunocompromised patients.

We found that there is a wide variation in HEV infection definition in literature, although in accordance with a recent surveillance report by the European Centre for Disease Prevention and Control (ECDC), concerning hepatitis E virus in Europe, a positive case is considered in a patient with an HEV RNA positive sample (serum, plasma, blood or feces) or both anti-HEV IgM and IgG positive sample [12]. HEV RNA is detected usually between 2 and 8 weeks when viremia reaches its peak before strong declining, then, around 3 weeks after clinical symptoms, HEV RNA becomes undetectable, with the virus continuing to be shed in the stool for another 1-2 weeks. On the other hand, the anti-HEV IgM immune response remains detectable for 3-12 months and the IgG response reaches its peak four weeks later than IgM, remaining detectable for several years, although the exact duration of this response remains uncertain $[20,30]$.

The fact that there is no standardized case definition, in addition to the heterogeneity in the analytical sensitivity of the commercial HEV assays, makes the comparison of studies more difficult. In this systematic review, all the seven studies performed the detection of HEV RNA, the most robust marker of acute/active infection [18,21,35-39], while four performed also the detection of anti-HEV IgM/IgG $[18,35,37,38]$. The studies that performed HEV RNA detection as the diagnostic method for HEV infection in HSCT recipients revealed similar results between them, with an overall prevalence of HEV infection of $1.5 \%$. Furthermore, we found that the overall prevalence of antiHEV IgM was of $2.0 \%$, a comparable value to the reported by the detection of HEV RNA, which is expected since both are markers of acute infection.

The analysis of anti-HEV IgG showed an overall seroprevalence of $11.4 \%$ in HSCT patients, with significant differences between the results of the different studies, mainly due to the study from France which revealed a much higher prevalence when compared to the others studies [18]. These results are difficult to compare due to the differences in the sensitivities and specificities of anti-HEV IgG commercial immunoassays [40-43]. Moreover, seroprevalence is greatly influenced by food habits $[5,43]$ and to differences in culinary practices between countries/regions 43]. Reported anti-HEV IgG seroprevalence not only varies between countries but also within countries, with significant variance between regional areas 43]. Indeed, even seroprevalences in Europe are very difficult to compare in consequence of these serology limitations and very different rates have been reported across the continent, ranging from 1.3 to 52\% [44-46]. In Finland, anti-HEV IgG seroprevalence was found to be $27.6 \%$ in general population [47], while in Norway, Germany and Portugal reported seroprevalences were $11.4 \%, 16.8 \%$ and $16.3 \%$, respectively [48-50]. Furthermore, several countries such as Netherlands, Spain, France, and Southwest England have performed these studies in healthy blood donors reporting an antiHEV IgG prevalence ranging from 16.0 to $26.7 \%$ [51-54]. These facts contribute to the assumption that the anti-HEV IgG prevalence does not reflect the spread of HEV in the HSCT population, which reinforces the importance of better characterization in this group of patients.

HSCT recipients are at higher risk of HEV infection, that could lead to chronic infection, and the diagnosis of HEV infection is highly recommended. Over the last years, some attention has been given to this group of immunocompromised patients, but there is still a small number of studies in HSCT as demonstrated in the present systematic review. Therefore, more studies are needed to increase our understanding of the epidemiology of HEV in HSCT recipients.

\section{Funding}

This research did not receive any specific grant from funding agencies in the public, commercial, or not-for-profit sectors.

\section{Author contributions}

SC, acquisition of data, analysis and interpretation of data, drafting the article, final approval; CC, acquisition of data, final approval; CC and AT final approval; RM, conception and design of the study, final approval; MSJN, interpretation of data, revising the article, final

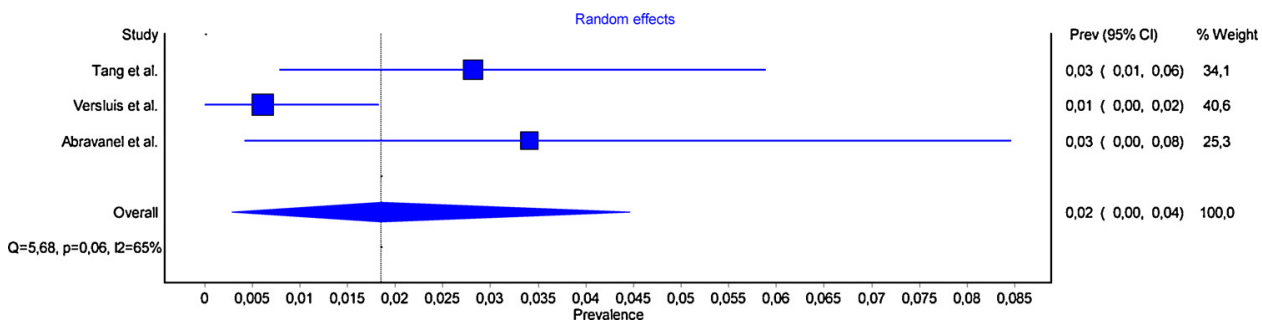

Fig. 3. Forest plot of the overall prevalence based on the detection of IgM anti-HEV.

Abbreviations: p, $p$-value; Prev (95\%CI), Prevalence 95\% Confidence Interval. 


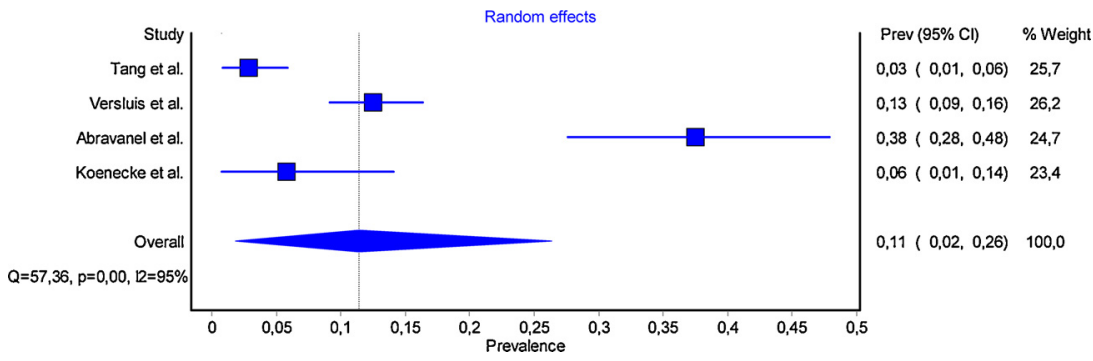

Fig. 4. Forest plot of the overall prevalence based on the detection of IgG anti-HEV.

Abbreviations: p, $p$-value; Prev (95\%CI), Prevalence 95\% Confidence Interval.

approval; HS, analysis and interpretation of data, conception and design of the study, revising the article, final approval.

\section{Declaration of Competing Interest}

The authors declare they have no conflict of interest.

\section{References}

[1] World Health Organization, Hepatitis E, (2018) Published September 19, 2018 Accessed March 22, 2019 https://www.who.int/news-room/fact-sheets/detail/ hepatitis-e.

[2] World Health Organization, Hepatitis E | Immunization, Vaccines and Biologicals, WHO, 2015 Published October 19, 2015. Accessed March 22, 2019 http://www. who.int/immunization/diseases/hepatitisE/en/.

[3] N. Kamar, R. Bendall, F. Legrand-Abravanel, et al., Hepatitis E, Lancet 379 (9835) (2012) 2477-2488, https://doi.org/10.1016/S0140-6736(11)61849-7.

[4] X.-J. Meng, Zoonotic and foodborne transmission of hepatitis E virus, Semin. Liver Dis. 33 (1) (2013) 41-49, https://doi.org/10.1055/s-0033-1338113.

[5] World Health Organization, The Global Prevalence of Hepatitis E Virus Infection and Susceptibility: a Systematic Review, (2010) https://apps.who.int/iris/handle/ 10665/70513.

[6] N. Pavio, X.-J. Meng, C. Renou, Zoonotic hepatitis E: animal reservoirs and emerging risks, Vet. Res. 41 (6) (2010) 46, https://doi.org/10.1051/vetres/2010018.

[7] H. Sonoda, M. Abe, T. Sugimoto, et al., Prevalence of hepatitis E virus (HEV) Infection in wild boars and deer and genetic identification of a genotype $3 \mathrm{HEV}$ from a boar in Japan, J. Clin. Microbiol. 42 (11) (2004) 5371-5374, https://doi, org/10.1128/JCM.42.11.5371-5374.2004.

[8] S. Tei, N. Kitajima, K. Takahashi, S. Mishiro, Zoonotic transmission of hepatitis E virus from deer to human beings, Lancet 362 (9381) (2003) 371-373, https://doi. org/10.1016/S0140-6736(03)14025-1.

[9] D. Huzly, M. Umhau, D. Bettinger, et al., Transfusion-transmitted hepatitis E in Germany, 2013, Euro Surveill. 19 (21) (2014).

[10] L. Hauser, A.-M. Roque-Afonso, A. Beylouné, et al., Hepatitis E transmission by transfusion of Intercept blood system-treated plasma, Blood 123 (5) (2014) 796-797, https://doi.org/10.1182/blood-2013-09-524348.

[11] P.E. Hewitt, S. Ijaz, S.R. Brailsford, et al., Hepatitis E virus in blood components: a prevalence and transmission study in southeast England, Lancet 384 (9956) (2014) 1766-1773, https://doi.org/10.1016/S0140-6736(14)61034-5.

[12] E.J. Aspinall, E. Couturier, M. Faber, et al., Hepatitis E virus infection in Europe: surveillance and descriptive epidemiology of confirmed cases, 2005 to 2015, Eurosurveillance 22 (26) (2017) 30561, https://doi.org/10.2807/1560-7917.ES. 2017.22.26.30561.

[13] H. Koot, B.M. Hogema, M. Koot, M. Molier, H.L. Zaaijer, Frequent hepatitis E in the Netherlands without traveling or immunosuppression, J. Clin. Virol. 62 (2015) 38-40, https://doi.org/10.1016/j.jcv.2014.11.020.

[14] C. Adlhoch, A. Avellon, S.A. Baylis, et al., Hepatitis E virus: assessment of the epidemiological situation in humans in Europe, 2014/15, J. Clin. Virol. 82 (2016) 9-16, https://doi.org/10.1016/j.jcv.2016.06.010.

[15] S. Ijaz, A.J. Vyse, D. Morgan, R.G. Pebody, R.S. Tedder, D. Brown, Indigenous hepatitis E virus infection in England: more common than it seems, J. Clin. Virol. 44 (4) (2009) 272-276, https://doi.org/10.1016/j.jcv.2009.01.005.

[16] S. Pischke, P. Behrendt, C.-T. Bock, W. Jilg, M.P. Manns, H. Wedemeyer, Hepatitis E in Germany-an under-reported infectious disease, Arztebl. Int. 111 (35-36) (2014) 577-583, https://doi.org/10.3238/arztebl.2014.0577.

[17] P. Chalupa, P. Vasickova, I. Pavlik, M. Holub, Endemic hepatitis e in the Czech Republic, Clin. Infect. Dis. 58 (4) (2014) 509-516, https://doi.org/10.1093/cid/ cit782.

[18] F. Abravanel, J.-M. Mansuy, A. Huynh, et al., Low risk of hepatitis E virus reactivation after haematopoietic stem cell transplantation, J. Clin. Virol. 54 (2) (2012) 152-155, https://doi.org/10.1016/j.jcv.2012.02.015.

[19] N. Kamar, H.R. Dalton, F. Abravanel, J. Izopet, Hepatitis e virus infection, Clin. Microbiol. Rev. 27 (2014), https://doi.org/10.1128/CMR.00057-13.

[20] N. Kamar, J. Izopet, N. Pavio, et al., Hepatitis E virus infection, Nat. Rev. Dis. Primers 3 (2017) 17086, https://doi.org/10.1038/nrdp.2017.86.

[21] M.J. Ankcorn, S. Ijaz, J. Poh, et al., Toward systematic screening for persistent hepatitis E virus infections in transplant patients, Transplantation 102 (7) (2018) 1139-1147, https://doi.org/10.1097/TP.0000000000002097.

[22] P. Ljungman, M. Bregni, M. Brune, et al., Allogeneic and autologous transplantation for haematological diseases, solid tumours and immune disorders: Current practice in Europe 2009, Bone Marrow Transplant. 45 (2) (2010) 219-234, https://doi.org/ 10.1038/bmt.2009.141.

[23] L. Galgano, D. Hutt, HSCT: how does It work? in: M. Kenyon, A. Babic (Eds.), The European Blood and Marrow Transplantation Textbook for Nurses: Under the Auspices of EBMT, Springer International Publishing, Cham, 2018, pp. 23-36, , https://doi.org/10.1007/978-3-319-50026-3_2.

[24] J. Storek, G. Espino, M.A. Dawson, B. Storer, M.E. Flowers, D.G. Maloney, Low Bcell and monocyte counts on day 80 are associated with high infection rates between days 100 and 365 after allogeneic marrow transplantation, Blood 96 (9) (2000) 3290-3293.

[25] E.-J. Wils, B. van der Holt, A.E.C. Broers, et al., Insufficient recovery of thymopoiesis predicts for opportunistic infections in allogeneic hematopoietic stem cell transplant recipients, Haematologica 96 (12) (2011) 1846-1854, https://doi.org/ 10.3324/haematol.2011.047696.

[26] E.-J. Wils, J.J. Cornelissen, Thymopoiesis following allogeneic stem cell transplantation: new possibilities for improvement, Blood Rev. 19 (2) (2005) 89-98, https://doi.org/10.1016/j.blre.2004.04.001.

[27] M.F. Leahy, K.M. Trentino, C. May, S.G. Swain, H. Chuah, S.L. Farmer, Blood use in patients receiving intensive chemotherapy for acute leukemia or hematopoietic stem cell transplantation: the impact of a health system-wide patient blood management program: impact of pbm program in hematology, Transfusion 57 (9) (2017) 2189-2196, https://doi.org/10.1111/trf.14191.

[28] M.A. Warner, N.S. Jambhekar, S. Saadeh, et al., Implementation of a patient blood management program in hematopoietic stem cell transplantation: PBM in stem cell transplantation, Transfusion (June 2019) (2019), https://doi.org/10.1111/trf. 15414.

[29] A. Singh, R. Seth, A. Gupta, et al., Chronic hepatitis E - an emerging disease in an immunocompromised host, Gastroenterol. Rep. (Oxf) 6 (2) (2018) 152-155, https://doi.org/10.1093/gastro/gow024.

[30] G.W. Webb, H.R. Dalton, Hepatitis E: an underestimated emerging threat, Ther. Adv. Infect. Dis. 6 (2019) 204993611983716, , https://doi.org/10.1177/ 2049936119837162.

[31] S.D. Pas, R.A. de Man, C. Mulders, et al., Hepatitis E virus infection among solid organ transplant recipients, the Netherlands, Emerging Infect. Dis. 18 (5) (2012) 869-872, https://doi.org/10.3201/eid1805.111712.

[32] N. Kamar, J. Selves, J.-M. Mansuy, et al., Hepatitis E virus and chronic hepatitis in organ-transplant recipients, N. Engl. J. Med. 358 (8) (2008) 811-817, https://doi. org/10.1056/NEJMoa0706992.

[33] E.B. Haagsma, A.P. van den Berg, R.J. Porte, et al., Chronic hepatitis E virus infection in liver transplant recipients, Liver Transpl. 14 (4) (2008) 547-553, https:// doi.org/10.1002/1t.21480.

[34] J.J. Barendregt, S.A. Doi, Y.Y. Lee, R.E. Norman, T. Vos, Meta-analysis of prevalence, J. Epidemiol. Community Health 67 (11) (2013) 974-978, https://doi.org/ 10.1136/jech-2013-203104.

[35] F.-F. Tang, X.-D. Mo, Y. Wang, et al., Hepatitis E virus infection after haploidentical haematopoietic stem cell transplantation: Incidence and clinical course, Br. J. Haematol. 184 (5) (2019) 788-796, https://doi.org/10.1111/bjh.15672.

[36] S.B. Willemse, D.L. Bezuur, P. Blom, et al., Hepatitis E virus infection and hepatic GvHD in allogeneic hematopoietic stem cell transplantation recipients, Bone Marrow Transplant. 52 (4) (2017) 622-624, https://doi.org/10.1038/bmt.2016. 327.

[37] J. Versluis, S.D. Pas, H.J. Agteresch, et al., Hepatitis E virus: an underestimated opportunistic pathogen in recipients of allogeneic hematopoietic stem cell transplantation, Blood 122 (6) (2013) 1079-1086, https://doi.org/10.1182/blood-201303-492363.

[38] C. Koenecke, S. Pischke, A. Heim, et al., Chronic hepatitis E in hematopoietic stem cell transplant patients in a low-endemic country? Transpl. Infect. Dis. 14 (1) (2012) 103-106, https://doi.org/10.1111/j.1399-3062.2011.00702.x.

[39] I. Reekie, D. Irish, S. Ijaz, et al., Hepatitis E infection in stem cell and solid organ transplantpatients: A cross-sectional study: The importance of HEV RNA screening in peri-transplant period, J. Clin. Virol. 107 (2018) 1-5, https://doi.org/10.1016/j. jcv.2018.07.011.

[40] R. Bendall, V. Ellis, S. Ijaz, R. Ali, H. Dalton, A comparison of two commercially available anti-HEV IgG kits and a re-evaluation of anti-HEV IgG seroprevalence data in developed countries, J. Med. Virol. 82 (5) (2010) 799-805, https://doi.org/10. 
1002/jmv.21656.

[41] S.D. Pas, R.H.R.A. Streefkerk, M. Pronk, et al., Diagnostic performance of selected commercial HEV IgM and IgG ELISAs for immunocompromised and immunocompetent patients, J. Clin. Virol. 58 (4) (2013) 629-634, https://doi.org/10. 1016/j.jcv.2013.10.010.

[42] A. Avellon, L. Morago, M. Garcia-Galera del Carmen, M. Munoz, J.-M. Echevarría, Comparative sensitivity of commercial tests for hepatitis E genotype 3 virus antibody detection, J. Med. Virol. 87 (11) (2015) 1934-1939, https://doi.org/10.1002/ jmv.24251.

[43] J. Hartl, B. Otto, R.G. Madden, et al., Hepatitis E Seroprevalence in Europe: A metaanalysis, Viruses 8 (8) (2016), https://doi.org/10.3390/v8080211.

[44] D. Lapa, M.R. Capobianchi, A.R. Garbuglia, Epidemiology of hepatitis E virus in european countries, Int. J. Mol. Sci. 16 (10) (2015) 25711-25743, https://doi.org/ 10.3390/ijms161025711.

[45] G. Scotto, D. Martinelli, M. Centra, et al., Epidemiological and clinical features of HEV infection: a survey in the district of Foggia (Apulia, Southern Italy), Epidemiol. Infect. 142 (2) (2014) 287-294, https://doi.org/10.1017/S0950268813001167.

[46] J.-M. Mansuy, R. Bendall, F. Legrand-Abravanel, et al., Hepatitis E virus antibodies in blood donors, France, Emerging Infect. Dis. 17 (12) (2011) 2309-2312, https:// doi.org/10.3201/eid1712.110371.

[47] T. Kantala, L. Maunula, C.-H. von Bonsdorff, J. Peltomaa, M. Lappalainen, Hepatitis E virus in patients with unexplained hepatitis in Finland, J. Clin. Virol. 45 (2) (2009) 109-113, https://doi.org/10.1016/j.jcv.2009.03.006.

[48] I.B. Olsøy, S. Henriksen, F.H. Weissbach, et al., Seroprevalence of hepatitis E virus
(HEV) in a general adult population in Northern Norway: The Tromsø study, Med. Microbiol. Immunol. (March 2019) (2019), https://doi.org/10.1007/s00430-01900599-5.

[49] M.S. Faber, J.J. Wenzel, W. Jilg, M. Thamm, M. Höhle, K. Stark, Hepatitis E virus seroprevalence among adults, Germany, Emerging Infect. Dis. 18 (10) (2012) 1654-1657, https://doi.org/10.3201/eid1810.111756.

[50] M.S.J. Nascimento, S.S. Pereira, J. Teixeira, et al., A nationwide serosurvey of hepatitis E virus antibodies in the general population of Portugal, Eur. J. Public Health 28 (4) (2018) 720-724, https://doi.org/10.1093/eurpub/ckx213.

[51] E. Slot, B.M. Hogema, A. Riezebos-Brilman, T.M. Kok, M. Molier, H.L. Zaaijer, Silent hepatitis E virus infection in Dutch blood donors, 2011 to 2012, Euro Surveill. 18 (31) (2013).

[52] S. Sauleda, E. Ong, M. Bes, et al., Seroprevalence of hepatitis E virus (HEV) and detection of HEV RNA with a transcription-mediated amplification assay in blood donors from Catalonia (Spain), Transfusion 55 (5) (2015) 972-979, https://doi. org $/ 10.1111 / \operatorname{trf} .12929$

[53] J.M. Mansuy, P. Gallian, C. Dimeglio, et al., A nationwide survey of hepatitis E viral infection in French blood donors, Hepatology 63 (4) (2016) 1145-1154, https:// doi.org/10.1002/hep.28436.

[54] H.R. Dalton, W. Stableforth, P. Thurairajah, et al., Autochthonous hepatitis E in Southwest England: Natural history, complications and seasonal variation, and hepatitis E virus IgG seroprevalence in blood donors, the elderly and patients with chronic liver disease, Eur. J. Gastroenterol. Hepatol. 20 (8) (2008) 784-790, https://doi.org/10.1097/MEG.0b013e3282f5195a. 